\title{
Performance of the ATLAS Tile Calorimeter
}

\author{
Gabriele BERTOLI on behalf of the ATLAS collaboration* \\ Stockholm University (SE) \\ E-mail: gabriele.bertoliecern.ch
}

The Tile Calorimeter (TileCal) of the ATLAS experiment at the LHC is the central hadronic calorimeter designed for energy reconstruction of hadrons, jets, tau-particles and missing transverse momentum giving essential contribution to reconstructed objects and physics results. Tile$\mathrm{Cal}$ is a scintillator-steel sampling calorimeter and it covers the region $|\eta|<1$.7. Results on the calorimeter operation and performance are presented, including the calibration, stability, absolute energy scale, and uniformity. These results show that the TileCal performance is within the design requirements.

XXVII International Symposium on Lepton Photon Interactions at High Energies

17-22 August 2015

Ljubljana, Slovenia

${ }^{*}$ Speaker. 


\section{Introduction}

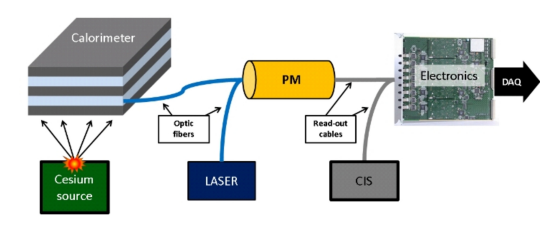

Figure 1: The ATLAS Tile calorimeter calibration chain.

The ATLAS Tile calorimeter[1] is the central hadronic calorimeter of ATLAS. The analog signals from the photomultipliers tubes (PMTs) are amplified using a bi-gain system, with low and high gains (LG and HG). The LG analog signals are sent to L1 trigger system. Both LG and HG are digitized and sent to the precision calorimeter read out. The TileCal front-end electronics read out the signals produced by about 10000 channels that correspond to approximately 5000 physical cells. The read-out system is responsible for reconstructing the data in real-time. The digitized signals are reconstructed with the Optimal Filtering algorithm, which computes for each channel the signal amplitude, time and quality factor at the required high rate (up to $100 \mathrm{kHz}$ ). Each stage of the signal production from scintillation light to the signal reconstruction is monitored and calibrated.

\section{Calibration chain}

The energy deposited in the TileCal cells is initially measured in ADC counts, but for physics analysis it needs to be converted into $\mathrm{GeV}$ following the equation: $E[\mathrm{GeV}]=A[A D C] \times C_{A D C \rightarrow p C} \times$ $C_{\text {laser }} \times C_{C s} \times C_{p C \rightarrow G e V}$ where $A[A D C]$ is the measured channel signal amplitude and $C_{p C \rightarrow G e V}$ is a constant to convert the charge to electromagnetic scale and is measured during test beam using electrons. The other conversion factors are determined using dedicated systems, the charge injection system (CIS) allows to measure the $C_{A D C \rightarrow p C}$ conversion factor, the laser system determines the $C_{\text {laser }}$ constant while the Cesium system gives the $C_{C s}$. Figure 1 sketches the calibration chain.

\subsection{The charge injection system}

The CIS is used to calibrate the read out electronics by injecting a known charge and measuring the resulting response of the electronics. This allows to determine the conversion factor from $p C$ to $A D C$ counts $\left(C_{A D C \rightarrow p C}\right)$. The conversion factor over the period shown in Figure 2a is stable at the per-mil level. Channels not responding to CIS or exhibiting large fluctuations are not considered, these constitutes approximately $1 \%$ of all the channels. The RMS value arises from typical fluctuations during calibration.

\subsection{Laser calibration}

The laser system main purpose is to monitor the photomultipliers tubes and the downstream electronics. Well calibrated light pulses are sent to the PMTs and by reconstructing the signal it is possible to extract the PMTs' gain. We can see from Figure $2 \mathrm{~b}$ that the stability of the upgraded laser system (better than $1 \%$ over a month) is within expectation. The PMTs response is normalized to the first dataset and the RMS relative to the mean ratio is $5.18 \times 10^{-3}$ over a one month period. 


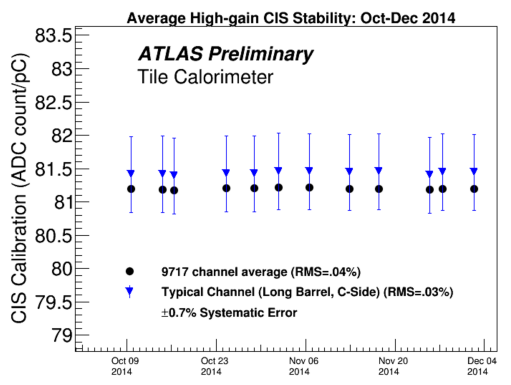

(a) TileCal-wide CIS calibration constant averages of all the high-gain channels for each CIS calibration run from 01 Oct 2014 to 04 Dec 2014 [2].

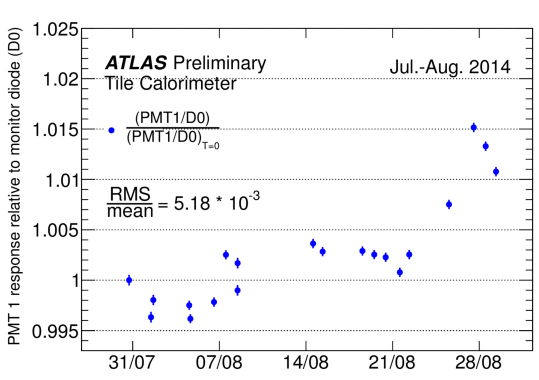

(b) Normalized response of a TileCal PMT illuminated with the upgraded laser calibration system, as function of time [2].

Figure 2

\subsection{The cesium system}

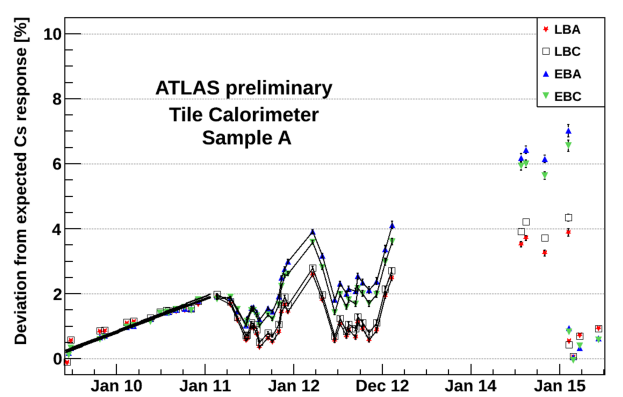

Figure 3: Variation of Tile Calorimeter response over period of 6 years measured in Cesium calibration runs averaged over all cells in a given sample. The big gap between February 2013 and July 2014 corresponds to the maintenance campaign during long shutdown [2].

The cesium calibration measures the response of the signal chain starting at the level of the scintillators in actual calorimeter cell. A movable radioactive source is circulated through all the detector cells to equalize their response. The signal generated by the Cs source is read out through a special "integrator" read out that integrates the analog PMT signals. In 2015 the high voltage $(\mathrm{HV})$ was adjusted to equalize the response of the cells so that the cesium conversion factor $\left(C_{C s}\right)$ would be 1. This can be seen in Figure 3 as the last points (25th February 2015) are close to zero and the spread in the detector barrels is small.

\section{Electromagnetic scale calibration}

The electromagnetic scale is studied by evaluating the ratio $d E / d l$ representing the energy deposited per unit length by muons in TileCal. The muons can either come from cosmic rays or from heavy gauge boson decays in proton-proton collisions.

\subsection{Performance studies using cosmic runs}

Figure 4a shows the response of the long barrel BC cells as a function of the track path length $d l$ to selected cosmic muons. The excess of events visible around $840 \mathrm{~mm}$ is due to the geometry 


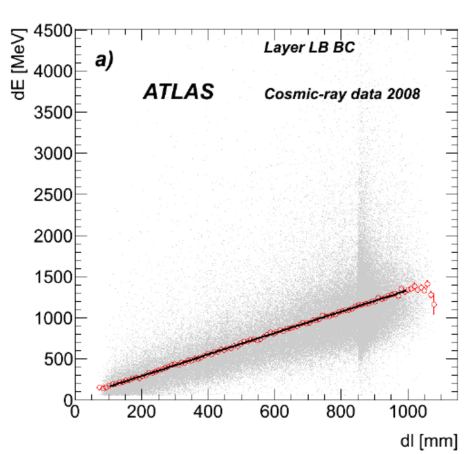

(a) Response of the barrel module LB-BC cells as a function of track path length obtained using 2008 data [3].

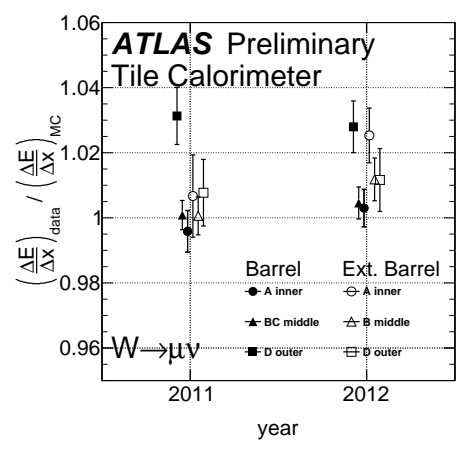

(b) Data to Monte Carlo comparison of the $(\Delta E / \Delta x)_{\mathrm{data}} /(\Delta E / \Delta x)_{\mathrm{MC}}$ ratio for collision muons for two different years. [2].

Figure 4

of the $\mathrm{BC}$ cells. The superimposed linear fit shows that the energy deposited by the muons in the cells scales linearly with the track path length.

\subsection{Performance using proton-proton collision}

The detector energy response can also be evaluated using proton-proton collisions. To this end, single track muons produced in association with a neutrino coming from a $W$ boson $(W \rightarrow \mu v)$ are selected using cuts in missing transverse momentum $E_{\mathrm{T}}^{\text {miss }}>50 \mathrm{GeV}$ and transverse mass $M_{\mathrm{T}}>25 \mathrm{GeV}$. Figure $4 \mathrm{~b}$ shows the response $(\Delta E / \Delta x)_{\text {data }} /(\Delta E / \Delta x)_{\mathrm{MC}}$ to these muons for each layer; this is calculated as the weighted average in $\eta$ for each cell type for both data and Monte Carlo simulation, gap/crack scintillators and cells with $\eta<0.1$ are excluded from the analysis. It confirms the uniformity and stability of the EM scale in Run 1.

\section{Performance using proton-proton collisions}

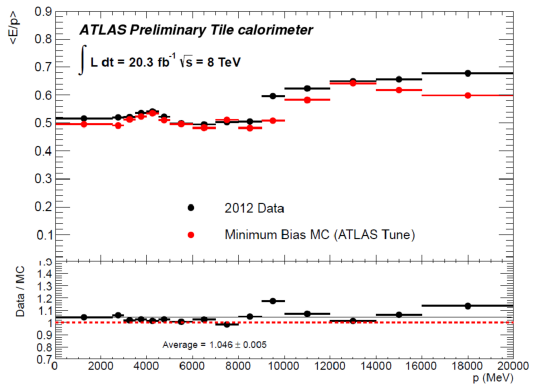

(a) Calorimeter Response characterized by energy over momentum (E/p) for isolated tracks, as measured with the Tile Calorimeter, using proton-proton collision data from 2012 in the Minimum Bias stream [2].

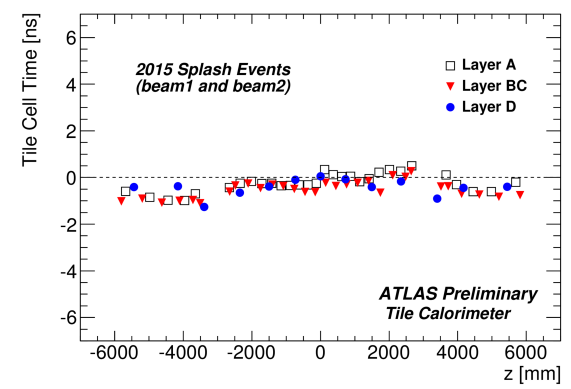

(b) Timing of ATLAS TileCal signals recorded with single beam data on April 2015 [4]. 
The energy response of the calorimeter can also be evaluated using charged hadrons. The energy deposit of isolated tracks compatible with minimum ionizing particles in the calorimeter, $E$, is compared with the momentum measured by the inner detector, $p$, in data and Monte Carlo (MC) simulations. Figure 5a shows the average $E / p$ ratio for single hadrons as a function of the momentum $p$, integrated over the pseudo-rapidity, $\eta$ and $\phi$ coverage of the Tile calorimeter. The largest discrepancy is $18 \%$ for $9<p<10 \mathrm{GeV}$.

\section{Timing calibration}

The LHC can produce so called beam splashes, by aiming protons at a collimator upstream of the detector. This results in a large number of particles essentially lighting up every cell of the calorimeter. In 2015 such events were used to equalize the timing of the TileCal. Time of flight measurements are used to discriminate against relativistic particles in the search for new physics, it is ATLAS convention to assign time $=0$ to particles traveling at the speed of light from the interaction point. Figure $5 \mathrm{~b}$ shows the time average cells with same $\phi$ as function of the zcoordinate along the beam axis. Within $1.5 \mathrm{~ns}$, after corrections from laser data and time-of-flight, the initial time equalization has a flat distribution.

\section{Conclusion}

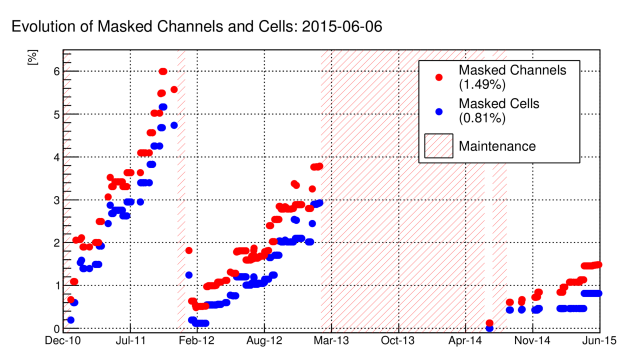

Figure 6: Evolution of the fraction of masked TileCal cells and channels as a function of time starting from December 2010; the large gap between March 2013 and April 2014 represents the long maintenance shutdown. [5].

The calibration systems of the ATLAS Tile calorimeter have been presented. The response from the calibration chain is stable. The CIS conversion factor is stable at the per mil level over the period from 09 October 2014 to 04 December 2014; the upgraded laser system stability is better than $1 \%$ over a month period and the cesium variation response during the second round of equalization (25th February 2015) is minimum. Performance studies using proton-proton collisions and cosmic runs, show that the detector operation is robust. Initial time calibration distribution in 2015 splash events is flat within $1.5 \mathrm{~ns}$ after laser and time-of-flight corrections. After the long shutdown all known problems have been solved and, as can be seen from Figure 6, the current number of masked cells and channels is less than $2 \%$. Overall, TileCal performs within expectations and together with other ATLAS sub-detectors it contributes to the measurement of jets, tau and missing energy. 


\section{References}

[1] The ATLAS Collaboration. "The ATLAS Experiment at the CERN Large Hadron Collider". In: Journal of Instrumentation 3.08 (2008), S08003. URL: http: / / stack s . iop . org / $1748-0221 / 3 / i=08 / a=S 08003$.

[2] TileCal Public Results. URL: https : / / twiki . cern . ch / twiki / bin/view / AtlasPublic/TileCaloPublicResults.

[3] The ATLAS Collaboration. "Readiness of the ATLAS Tile Calorimeter for LHC collisions". English. In: The European Physical Journal C 70.4 (2010), pp. 1193-1236. ISSN: 1434-6044. DOI: $10.1140 / \mathrm{epjc} / \mathrm{s} 10052-010-1508-y$. URL: http: / / dx.doi.org/10 . $1140 /$ epjc/s10052-010-1508-y.

[4] TileCal Approved Plots Timing. URL: https://twiki.cern.ch/twiki/bin/view/ AtlasPublic/ApprovedPlotsTileTiming.

[5] TileCal Approved Plots. URL: https : / / twiki . cern . ch / twiki / bin/view / AtlasPublic/ApprovedPlotsTile. 